\title{
EVALUACIÓN DE VISCOSIDAD Y COLOR EN LA PULPA DE MANGO COMÚN (Mangifera indica L) TRATADA ENZIMÁTICAMENTE
}

\section{EVALUATION OF THE VISCOSITY AND THE COLOR OF COMMON MANGO PULP (Mangifera indica L.) ENZYMATICALLY TREATED}

\author{
Víctor D. Quintero C. ${ }^{1}$, Alba L. Duque C. ${ }^{2}$, Germán A. Giraldo G. ${ }^{3}$ \\ Recibido para publicación: Febrero 17 de 2012 - Aceptado para publicación: Agosto 27 de 2012
}

\begin{abstract}
RESUMEN
El mango es una de las frutas más importantes desde el punto de vista comercial debido a que gracias a sus excelentes características sensoriales se pueden obtener una serie de productos derivados, entre ellos la pulpa. El objetivo de este trabajo fue estudiar la acción de la pectinasa sobre la estabilización de la pulpa de mango común. En este trabajo se obtuvo un producto homogenizado de mango común fresco, a partir del licuado de la pulpa cruda y un filtrado a través de una malla de $100 \mu \mathrm{m}$ de tamaño de poro. Este homogenizado se caracterizó y se comparó con los valores medidos de una pulpa de fruta comercial y un néctar comercial. Posteriormente se procedió a realizarle un tratamiento enzimático con pectinasa comercial con el fin de disminuir la viscosidad del homogenizado variando la concentración de la enzima, el tiempo y la temperatura de tratamiento. Los datos fueron procesados con ayuda de la metodología de superficies de respuesta apoyados en el software statgraphics centurion XVI. La pulpa inicial tuvo una viscosidad de 2.419,4 \pm 48,4 mPa*s y la pulpa y el néctar comerciales de 477,6 y 54,0 mPa*s respectivamente. Los resultados obtenidos muestran que para que el homogenizado tenga la viscosidad de una pulpa comercial es necesario realizar un tratamiento enzimático a $35^{\circ} \mathrm{C}$ por 90 minutos y con una concentración de pectinasa de $0,1 \%$ y para obtener un homogenizado con viscosidad similar a la de un néctar se necesita un tratamiento a $30^{\circ} \mathrm{C}$ por 85,5 minutos y con una concentración de pectinasa de $0,1 \%$.
\end{abstract}

Palabras clave: mango, pulpa, color, viscosidad.

\footnotetext{
${ }^{1}$ Químico, Especialista en Gerencia, Especialista en Docencia Universitaria, Magister en Química con énfasis en alimentos, Facultad de Ciencias Agroindustriales. Universidad del Quindío. Cra 15 \#12N Edificio de Ciencias Básicas, Laboratorio de Diseño de Nuevos Productos, Armenia, Quindío. victordumar@uniquindio.edu.co

${ }^{2}$ Licenciada en Química, Especialista en pos cosecha, Magister en Química con énfasis en alimentos, Facultad de Ciencias Básicas. Universidad del Quindío.

${ }^{3}$ Licenciado en Química. Magister en Nutrición, Doctor en ciencia y tecnología de alimentos, Facultad de Ciencias Agroindustriales. Universidad del Quindío.
} 


\begin{abstract}
The mango is one of the most important fruit from the commercial point of view because to its excellent sensory characteristics can get a number of products, including pulp. The aim of this work was to study the action of pectinase on stabilizing the common mango pulp. In this work yielded a product homogenized of fresh mango from a liquefied raw flesh and filter through a grid of $100 \mu \mathrm{m}$ pore size. This homogenized was characterized and compared with the measured values of a pulp commercial fruit and commercial nectar. Then proceeded to perform commercial pectinase enzyme treatment to reduce the viscosity of the homogenized by varying the enzyme concentration, time and temperature treatment. Data were processed using response surface methodology supported by software STATGRAPHICS CENTURION XVI. The initial pulp contained a viscosity of 2.419, $4 \pm 48.4 \mathrm{mPa}^{*} \mathrm{sec}$ and the commercial pulp and Nectar 477,6 and 54,0 MPA* sec respectively. The results shows that the homogenized needs a treatment to $35^{\circ} \mathrm{C}$ for 90 minutes with pectinase $0.1 \%$ to obtain commercial pulp viscosity, and needs a treatment to $30^{\circ} \mathrm{C}$ during $85.5 \mathrm{~min}$. with pectinase $0,1 \%$ to obtain a homogenized similar to a commercial nectar.
\end{abstract}

Key words: mango, pulp, color and viscosity.

\section{INTRODUCCIÓN}

El mango (Mangifera indica L) es la fruta comercial de mayor disponibilidad en países de la región tropical de América (Pelegrine et al. 2002). El mango común tiene como características principales su contenido de pectina, azúcar, sabor, aroma y color que lo hacen atractivo para el consumidor; además del alto contenido de fibra insoluble comparado con otras variedades de mango (Bhattacharya y Rastogi 1998). En la región central de Colombia existen productores de mango, que lo comercializan en fresco; sin embargo, los problemas nacen en la superproducción, al no lograr vender todo lo producido y las pérdidas debido a la mala manipulación del producto y al daño producido por factores externos como insectos, aves, bacterias, etc. Todo esto da alrededor de un $40 \%$ en pérdidas poscosecha de fruto (Liew et al. 2007), por esto es indispensable plantear soluciones de cómo aumentar la vida útil de este fruto.

El mango común (Mangiferaindica L) es utilizado industrialmente para la producción de pulpas, concentrados, néctares, jugos, mermelada y flakes (Sreenath et al. 1995). Algunos productos derivados del procesamiento del mango guardan características organolépticas semejantes al original, estimulando una alta aceptabilidad en el mercado (Kashyap et al. 2001). Los productos elaborados a partir del mango de mayor demanda en el mercado son las pulpas y los jugos que se obtienen homogenizando el fruto y posteriormente se despulpa y se filtra (Alkorta et al. 1998; Ceci y Lozano 1998). A los productos derivados del mango se les realizan procedimientos de reducción de viscosidad debido a su alta concentración de pectinas. El procedimiento más ampliamente utilizado para tal fin es la aplicación de enzimas pectoliticas que provocan una degradación de las paredes celulares de la pulpa cruda del mango por hidrólisis de las pectinas, con lo cual se disminuye la viscosidad del homogenizado, además ayuda a aumentar el rendimiento en la elaboración de jugos y néctares (Alkorta et al. 1998). Las enzimas pécticas comerciales, o 
pectinasas han sido usadas en la manufactura de jugos de frutas para despectinizarlos, provocando una disminución de la turbidez y previniendo la formación de precipitados.

Las preparaciones de pectinasas comerciales están disponibles en mezclas de pectinestearasa, poligalacturonasa y pectinliasa. El completo rendimiento de la ruptura de las pectinas se puede asegurar si los tres tipos de enzimas están presentes en las proporciones adecuadas, además de la determinación de las temperaturas óptimas de trabajo para cada mezcla de enzimas para cada tipo de sustrato. Las pectianasas comerciales se caracterizan por tener un amplio espectro de aplicación en la industria de los jugos, pero al igual se necesita estandarizar completamente el proceso determinando los parámetros claves de reacción como lo son tiempo, temperatura, concentración de enzima, velocidad de agitación y tipo de sustrato. (Quaglia y Gennaro 2003)

El objetivo principal de este trabajo es evaluar el efecto de la pactinasa sobre el cambio de color y viscosidad en la pulpa de mango común (Manguifera indical L.) tomando como variables de proceso la temperatura de tratamiento, la concentración de la enzima y el tiempo de reacción).

\section{MATERIALES Y MÉTODOS}

\section{Material Vegetal}

Los frutos de mango común (Manguifera indical L.) se adquirieron en la central mayorista de acopio de frutas de la ciudad de Armenia con aproximadamente cuatro horas de cosechados los cuales se encontraban en estado de madurez fisiológica, en donde el fruto no ha desarrollado todos sus atributos de calidad, pero al cosecharse adquiere sus características organolépticas. Posteriormente en el laboratorio de Diseño de nuevos productos de la Universidad del Quindío se almacenaron a temperatura ambiente por tres días con el fin de que adquirieran un porcentaje de sólidos solubles entre 13 y $16 \%$.

\section{Obtención del homogenizado}

El mango se lavó con hipoclorito de sodio, se escaldó a $75^{\circ} \mathrm{C}$ durante 1 minuto, se peló, troceó y posteriormente se homogenizó en una licuadora convencional marca Osterizer durante 90 segundos a temperatura ambiente (Liew et al. 2007).

\section{Tratamiento Enzimático}

La enzima utilizada fue una Pectinasa de marca Naturalzyme ${ }^{\circledR}$ 40X. Se elaboró un diseño factorial de $3 * 3 * 3$ donde se evaluaron por triplicado el comportamiento de tres concentraciones de la enzima $(0,05 \%, 0,075 \%$ y $0,1 \%$ ), tres tiempos (30, 60 y 90 minutos) y tres temperatura de tratamiento $\left(30,35\right.$ y $\left.40^{\circ} \mathrm{C}\right)$ (Alkorta et al. 1998; Demir et al. 2001) sobre 250 gramos de mango homogenizado para un total de 81 tratamientos y como variables de respuesta se determinaron la viscosidad y el color. La temperatura fue controlada con ayuda de un baño termostatado, la agitación fue constante a aproximadamente $500 \mathrm{rpm}$. La enzima después del tratamiento fue inactivada en un baño de agua a $80^{\circ} \mathrm{C}$ por 20 segundos (Sarioglu et al. 2001). El pH del sistema se estabilizó a 3,8 con adición de acido cítrico.

Posterior a esto se procedió a medir la viscosidad de los tratamientos con un viscosímetro rotacional marca Selecta de 
referencia ST 2010 a temperatura ambiente y el color se midió a través de los parámetros $\mathrm{L}$, a y b con un colorímetro Minolta CR-10 y se calculó la variable $\Delta \mathrm{E}$ para determinar el cambio de color en los tratamientos.

Simultáneamente se caracterizó una pulpa y un néctar de mango comercial determinando los parámetros de viscosidad y color, con el fin de tomarlos como valores de referencia.

Los datos se procesaron con ayuda de la metodología de superficies de respuesta (Liew et al. 2007; Rai et al. 2004) en el software statgraphics centurion XVI, con el fin de determinar los tiempos, las concentraciones de enzima y las temperaturas optimas para obtener un producto con viscosidad y color semejante a la pulpa y el néctar comercial.

\section{RESULTADOS Y DISCUSIÓN}

\section{Caracterización inicial}

En las Figuras 1 y 2 se observan las viscosidades y el color homogenizado de mango, la pulpa comercial y el néctar comercial. Se puede observar una diferencia significativa al 95\% de confiabilidad en ambos parámetros, esto es debido a las diferentes condiciones de procesamiento y a su uso, pues es bien sabido que las pulpas deben de tener un carácter más viscoso que un néctar con el fin de que se asemeje en un mayor porcentaje a la pulpa de mango sin ningún tratamiento. Se observó por las desviaciones obtenidas que las diferencias entre los tratamientos, tanto en la viscosidad como en el color, son significativas, quedando claro que para poder utilizar el homogenizado comercialmente hay que someterlo a un proceso tecnológico de reducción de viscosidad.

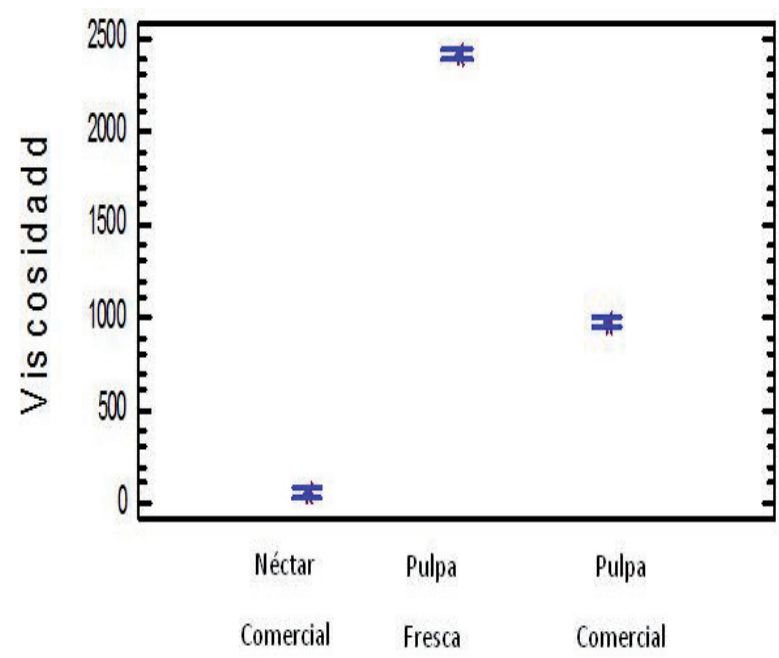

Figura 1. Comparación de las viscosidades (mPa*s) del néctar y pulpa comercial y la pulpa fresca.

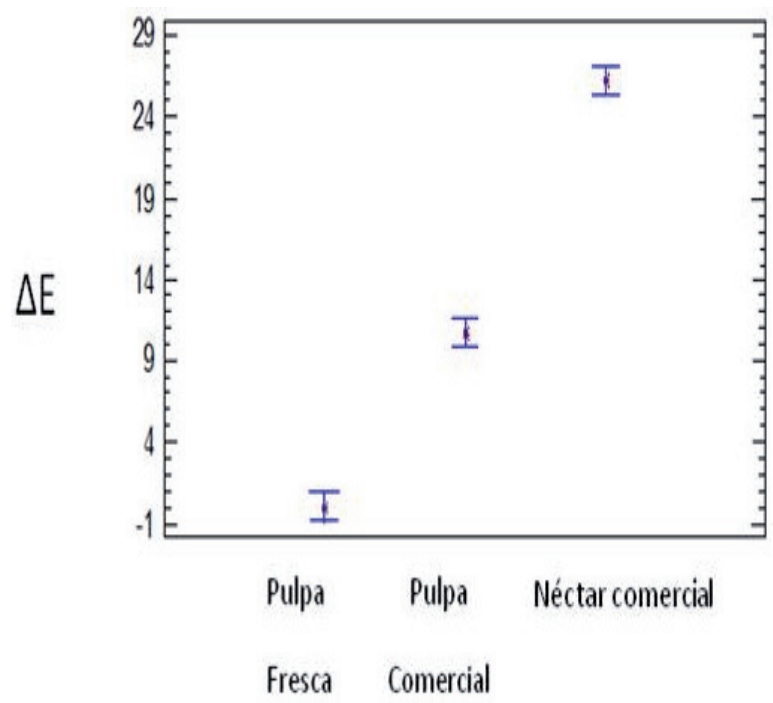

Figura 2. Comparación del cambio de color de del néctar y pulpa comercial y la pulpa fresca.

Para tal fin existen diferentes metodologías utilizadas industrialmente entre ellas están la ultrafiltración, la nanofiltración y la utilización de enzimas pectolíticas (Rai et al. 2004).

\section{Tratamiento enzimático}

\section{Viscosidad}

En la Figura 3 se observó la evolución de la viscosidad con respecto al cambio de la concentración de la enzima agregada con 
temperatura y tiempo constantes. A $0,1 \%$ de enzima agregada se observó una disminución de la viscosidad debido a que según el funcionamiento de una enzima entre mayor concentración de la enzima mayor será su acción sobre el sustrato, cuando los demás parámetros como la concentración de sustrato, $\mathrm{pH}$ y temperatura permanecen constantes. En este caso la acción de la enzima estuvo marcada por la disminución de la viscosidad debido a la hidrólisis de las pectinas del mango (Bhattacharya y Rastogi 1998; Sreenath et al. 1995 y Bon et al 2010). El mejor comportamiento de la enzima se observó a 90 minutos de tratamiento ya que en este tiempo se observó una mayor reducción de viscosidad a medida que va aumentando la concentración de la enzima. Esto se debe a que al existir una alta concentración enzima, aumenta la cantidad de sitios activos para reaccionar con las pectinas del mango. Esta relación es teóricamente lineal (Alkorta et al. 1998 y Ceci y Lozano 1998) y la grafica se detiene en el momento que no existan más moléculas de pectina para reaccionar, ya que todas fueron hidrolizadas a ácidos galacturónicos. Si se amplía la cantidad de enzima, se incrementa el número de sitios

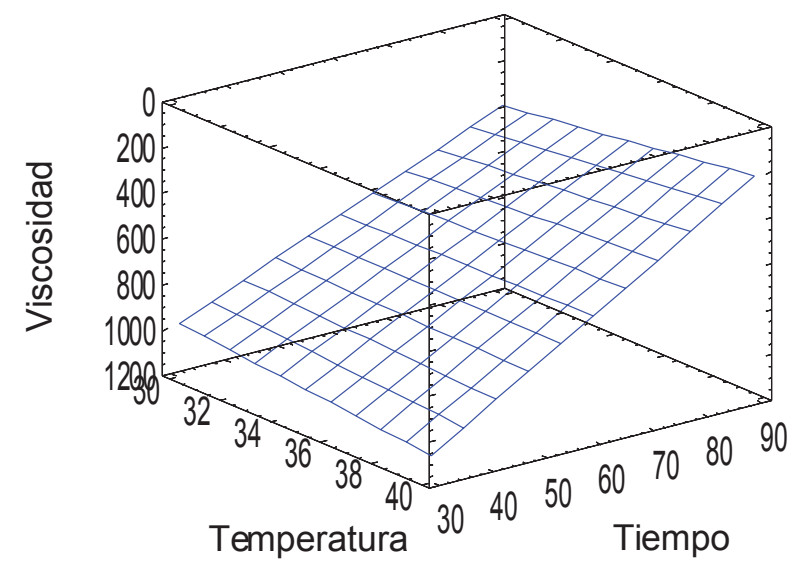

Figura 3. Diagrama de superficie de respuesta de tiempo, temperatura, viscosidad a 0,075\% de enzima. activos con lo cual se aumenta el número de moléculas de pectinas transformadas en ácidos galacturonicos libres, es decir, si se añade mas enzima, mayor será la transformación de las pectinas por tanto mayor será la disminución de la viscosidad del homogenizado de mango. Cuando la temperatura llega a un punto en el cual las cadenas polipeptidicas de la enzima toman una conformación tridimensional adecuada, se produce un mayor accionar de la enzima (Sarioglu et al 2001 y Alkorta et al 1998. En este trabajo solo se agrega enzima hasta un 0,1\%, ya que una concentración mayor provocaría una pérdida total de la viscosidad del homogenizado lo que afectaría las características fisicoquímicas propias de los productos a obtener, ya que las pulpas y los néctares tienen una viscosidad diferente a la de los fluidos acuosos como lo son los refrescos o los jugos diluidos de frutas, además de que industrialmente es inconveniente debido una alta concentración de enzima elevaría el costo del producto final. Algunos autores recomiendan la variación de la temperatura y el tiempo de tratamiento en lugar de aumentar drásticamente la concentración de la enzima, ya que la enzima puede aumentar su actividad a medida que llega a su temperatura óptima de acción sin importar la concentración de ésta (Demir et al. 2001; Sarioglu et al. 2001).

La viscosidad disminuye con respecto al tiempo de reacción en los tratamientos a 0,075\% y $0,1 \%$ de enzima. A $0,05 \%$ de enzima se observa un comportamiento muy variable. Con estas graficas se puede decir que la disminución de la viscosidad depende de la concentración de la enzima y que a mayor concentración de enzima mayor será el grado de transformación de los productos con respecto al tiempo independientemente de las temperaturas escogidas para este trabajo (Tabla 1). 
Tabla 1. Valores de viscosidad a diferentes tiempos, temperaturas y concentración de enzima.

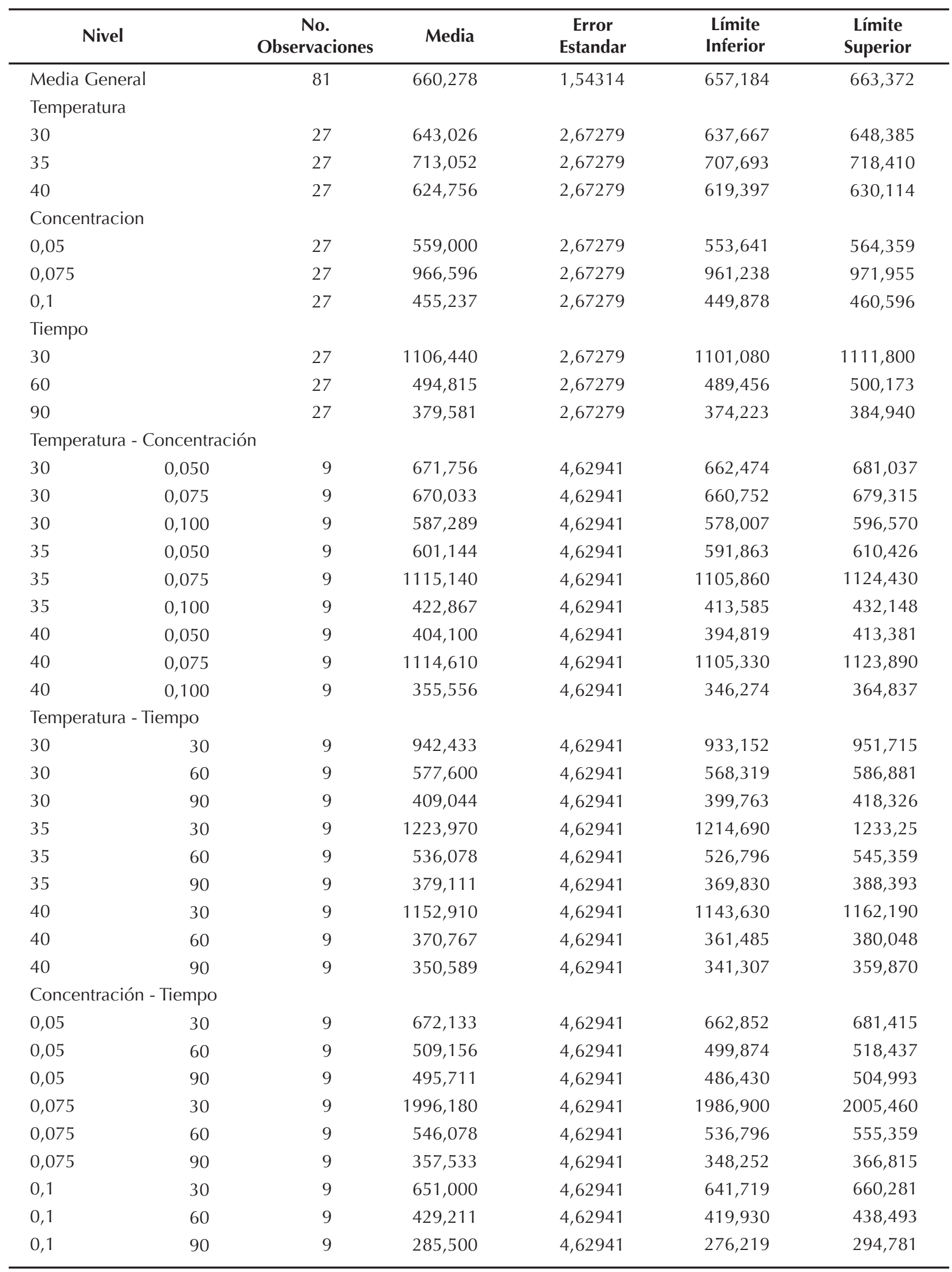


En la Tabla 1 se observa la disminución de la viscosidad con respecto a la temperatura sin importar la concentración de la enzima y el tiempo. En general se puede observar que a mayor temperatura existirá una mayor actividad de la enzima, pero en la elaboración de productos a base de materias primas biológicas se considera necesario no realizar tratamientos térmicos agresivos ya que estas pueden desnaturalizar proteínas, dañar las estructuras de las vitaminas y ayudar a la formación o degradación de compuestos químicos que pueden provocar cambios significativos, especialmente en el color (Alkorta et al. 1998; Ceci y Lozano 1998; Liew et al. 2007; Karunasawat and Anprung 2010; Valente y Ferrandis 2003). Por ello es recomendable disminuir las temperaturas de tratamiento y así proteger un poco el color original de la pulpa.

En el análisis del comportamiento de color se observaron cambios cuando se aumenta la concentración de la enzima en los tratamientos (Tabla 2), este efecto no es estadísticamente significativo según el análisis de varianza realizado, esto es coherente ya que no debe de existir ningún tipo de interacción química entre la enzima y los azucares reductores que provocan el pardeamiento no enzimático, o con los carotenoides que son los responsables del color amarillo propio de los mangos. Se puede observar en la mayoría de los tratamientos que a una temperatura de $40^{\circ} \mathrm{C}$ existe un mayor cambio de color del sistema, lo que indica que pueden aumentarse el número de reacciones de formación de compuestos oscuros (Karunasawat and Anprung 2010; Liew et al. 2007; Pretel et al. 1997; Valente y Ferrandis 2003).
La temperatura es una variable importante en el cambio de color debido a que a mayor temperatura, mayor será el cambio de color, aunque las diferencias entre los tratamientos seleccionados nos son estadísticamente significativas (P-value 0,5833), se puede observar que a $35^{\circ} \mathrm{C}$ ocurre el menor cambio de color, lo que indica que puede ser una buena temperatura de trabajo, ya que en los tratamientos térmicos aplicados a frutas o derivados se requiere que la materia prima cambie de una mínima forma con el fin de obtener un producto muy similar a la materia prima (Tabla 2).

Cuando se compara el cambio de color de los tratamientos con respecto al tiempo (Tabla 2) se observa una diferencia significativa entre el tratamiento a $90^{\circ} \mathrm{C}$ en el cual hay un cambio apreciable de color con respecto al cambio ocurrido a los $30^{\circ} \mathrm{C}$ (Grassin and Fauquembergue 1996; Valente, Bon et al 2010 y Ferrandis 2003). Esto indica que independientemente de la temperatura o la concentración de enzima que se trabaje, a medida que aumente el tiempo de tratamiento mayor será el cambio de color, lo que en el proceso de transformación de frutas es totalmente indeseable.

\section{Metodología de superficies de respuesta}

Cuando se comparan las variables concentración, temperatura y viscosidad en un diagrama de superficies de respuesta se observa claramente una disminución de la viscosidad del homogenizado (Figura 3).

De acuerdo a la Figura 3, la función obtenida se presenta en la ecuación 1.

$\mu=426,932+10,6921 * t+23,778 * T+$ 
Tabla 2. Valores del cambio de color $\Delta \mathrm{E}$ a diferentes tiempos, temperaturas y concentración de enzima.

\begin{tabular}{|c|c|c|c|c|c|c|}
\hline \multicolumn{2}{|c|}{ Nivel } & $\begin{array}{c}\text { No. } \\
\text { Observaciones }\end{array}$ & Media & $\begin{array}{c}\text { Error } \\
\text { Estandar }\end{array}$ & $\begin{array}{l}\text { Límite } \\
\text { Inferior }\end{array}$ & $\begin{array}{l}\text { Límite } \\
\text { Superior }\end{array}$ \\
\hline \multicolumn{2}{|c|}{ Media General } & 81 & 4,77778 & 0,0557883 & 4,66595 & 4,88963 \\
\hline \multicolumn{7}{|c|}{ Temperatura } \\
\hline \multicolumn{2}{|l|}{30} & 27 & 3,52963 & 0,0966281 & 3,33590 & 3,72336 \\
\hline \multicolumn{2}{|l|}{35} & 27 & 4,75556 & 0,0966281 & 4,56183 & 4,94928 \\
\hline \multicolumn{2}{|l|}{40} & 27 & 6,04815 & 0,0966281 & 5,85442 & 6,24188 \\
\hline \multicolumn{7}{|c|}{ Concentracion } \\
\hline \multicolumn{2}{|l|}{0,05} & 27 & 4,79259 & 0,0966281 & 4,59886 & 4,98632 \\
\hline \multicolumn{2}{|l|}{0,075} & 27 & 4,47037 & 0,0966281 & 4,27664 & 4,66410 \\
\hline \multicolumn{2}{|l|}{0,1} & 27 & 5,07037 & 0,0966281 & 4,87664 & 5,26410 \\
\hline \multicolumn{7}{|l|}{ Tiempo } \\
\hline \multicolumn{2}{|l|}{30} & 27 & 3,68519 & 0,0966281 & 3,49146 & 3,87891 \\
\hline \multicolumn{2}{|l|}{60} & 27 & 4,88889 & 0,0966281 & 4,69516 & 5,08262 \\
\hline \multicolumn{2}{|l|}{90} & 27 & 5,75926 & 0,0966281 & 5,56553 & 5,95299 \\
\hline \multicolumn{7}{|c|}{ Temperatura - Concentración } \\
\hline \multicolumn{2}{|c|}{$30 \quad 0,050$} & 9 & 5,41111 & 0,167365 & 5,07556 & 5,74666 \\
\hline 30 & 0,075 & 9 & 1,58889 & 0,167365 & 1,25334 & 1,92444 \\
\hline 30 & 0,100 & 9 & 3,58889 & 0,167365 & 3,25334 & 3,92444 \\
\hline 35 & 0,050 & 9 & 3,17778 & 0,167365 & 2,84223 & 3,51332 \\
\hline 35 & 0,075 & 9 & 5,32222 & 0,167365 & 4,98668 & 5,65777 \\
\hline 35 & 0,100 & 9 & 5,76667 & 0,167365 & 5,43112 & 6,10221 \\
\hline 40 & 0,050 & 9 & 5,78889 & 0,167365 & 5,45334 & 6,12444 \\
\hline 40 & 0,075 & 9 & 6,50000 & 0,167365 & 6,16445 & 6,83555 \\
\hline 40 & 0,100 & 9 & 5,85556 & 0,167365 & 5,52001 & 6,19110 \\
\hline Tempera & empo & & & & & \\
\hline 30 & 30 & 9 & 3,04444 & 0,167365 & 2,70890 & 3,37999 \\
\hline 30 & 60 & 9 & 3,77778 & 0,167365 & 3,44223 & 4,11332 \\
\hline 30 & 90 & 9 & 3,76667 & 0,167365 & 3,43112 & 4,10221 \\
\hline 35 & 30 & 9 & 3,56667 & 0,167365 & 3,23112 & 3,90221 \\
\hline 35 & 60 & 9 & 4,56667 & 0,167365 & 4,23112 & 4,90221 \\
\hline 35 & 90 & 9 & 6,13333 & 0,167365 & 5,79779 & 6,46888 \\
\hline 40 & 30 & 9 & 4,44444 & 0,167365 & 4,10890 & 4,77999 \\
\hline 40 & 60 & 9 & 6,32222 & 0,167365 & 5,98668 & 6,65777 \\
\hline 40 & 90 & 9 & 7,37778 & 0,167365 & 7,04223 & 7,71332 \\
\hline Concen & Tiempo & & & & & \\
\hline 0,05 & 30 & 9 & 4,26667 & 0,167365 & 3,93112 & 4,60221 \\
\hline 0,05 & 60 & 9 & 4,35556 & 0,167365 & 4,02001 & 4,69110 \\
\hline 0,05 & 90 & 9 & 5,75556 & 0,167365 & 5,42001 & 6,09110 \\
\hline 0,075 & 30 & 9 & 2,98889 & 0,167365 & 2,65334 & 3,32444 \\
\hline 0,075 & 60 & 9 & 5,14444 & 0,167365 & 4,80890 & 5,47999 \\
\hline 0,075 & 90 & 9 & 5,27778 & 0,167365 & 4,94223 & 5,61332 \\
\hline 0,1 & 30 & 9 & 3,80000 & 0,167365 & 3,46445 & 4,13555 \\
\hline 0,1 & 60 & 9 & 5,16667 & 0,167365 & 4,83112 & 5,50221 \\
\hline 0,1 & 90 & 9 & 6,24444 & 0,167365 & 5,90890 & 6,57999 \\
\hline
\end{tabular}


$1105,07 * C-0,516556 * t * T-94,9148 * t * C+$ $17,1778 * \mathrm{~T} * \mathrm{C}+0,911111 * \mathrm{t} * \mathrm{~T} * \mathrm{C} \quad$ EC. $\# 1$.

Donde:

$\mu=\operatorname{Viscosidad}\left(\mathrm{mPa}^{*} \mathrm{~s}\right)$

$\mathrm{C}=$ Concentración de enzima $(\%)$

$\mathrm{t}=$ Tiempo $(\min )$

$\mathrm{T}=$ temperatura $\left({ }^{\circ} \mathrm{C}\right)$

Con esta función se puede realizar una iteración para determinar las condiciones más adecuadas de temperatura, tiempo y concentración para obtener una pulpa y un néctar con viscosidad semejante a la de los productos comerciales (lagher et al. 2002; Liew et al. 2007; Rai et al. 2004).

En el caso de la pulpa en la cual la viscosidad del producto comercial es de 477,5 \pm 36,3 mPa*s, el resultado de la iteración indica que se debe trabajar a una concentración de enzima de 0,075\%, un tiempo de tratamiento de 75 minutos y una temperatura de $35^{\circ} \mathrm{C}$.

Para el caso del nectar comercial que tienen una viscosidad de 54,0 \pm 2,3 mPa*s, el resultado de la iteración dio como resultado una concentración de $0,1 \%$ de pectinasa un tiempo de tratamiento de 100,5 minutos a una temperatura de $35^{\circ} \mathrm{C}$.

Para realizar la iteracion anterior se tuvo en cuenta que la temperatura que mas influye en el cambio de color y en la disminución de la temperatura, por tanto se escogio $35^{\circ} \mathrm{C}$ que es una temperatura intermedia en la cual, no existe un cambio de color significativo y la viscosidad disminuye considerablemente.

Para el caso del diagrama de superficies de respuesta para el cambio de color se observa que el color cambia a medida que cambia la temperatura y el tiempo de tratamiento. (Figura 4).

Para el caso (Figura 4) del color la función que describe el comportamiento del cambio de color se ilustra en la ecuación 2.

$$
\begin{aligned}
& \Delta \mathrm{E}=-11,3646+1,07583 * \mathrm{~T}-0,018333 *^{*} \mathrm{t}- \\
& 191,278 * \mathrm{C}-0,01825 * \mathrm{~T} \wedge 2+0,00305556 * \mathrm{~T}^{*} \mathrm{t} \\
& +3,66667 * \mathrm{~T}^{*} \mathrm{C}-0,000581019 * \mathrm{t} \wedge 2+ \\
& 0,155556{ }^{*} \mathrm{t}^{*} \mathrm{C}+416,667^{*} \mathrm{C} \wedge 2 \quad \text { EC \# } 2
\end{aligned}
$$

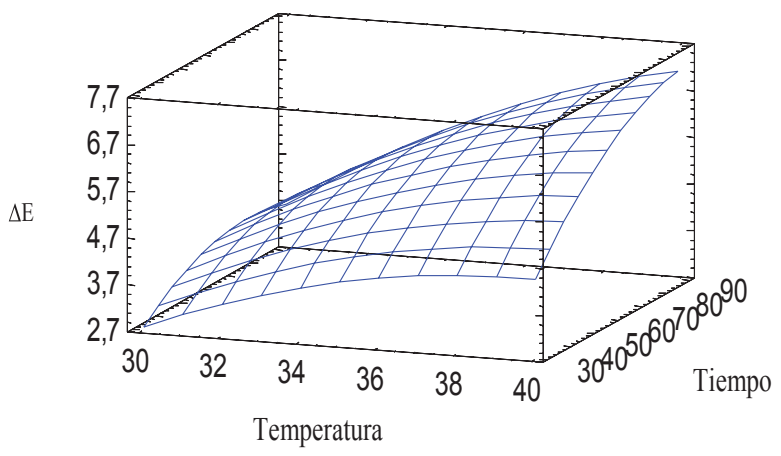

Figura 4. Diagrama de superficie de respuesta de tiempo, temperatura, cambio de color $\Delta \mathrm{E}$ a $0,075 \%$ de enzima.

La iteración con los datos anteriormente seleccionados (Tabla 3) indica que no hay un cambio drástico en el color, con lo que se pueden aceptar estas variables para la elaboración de pulpa y néctar con las características de viscosidad y color adecuadas para la elaboración de productos alimenticios comerciales. (Missang et al 1993, Pretel et al 1997 y Quaglia et al 2003).

Tabla 3. Valores obtenidos en la iteración sobre la ecuación 2 para obtención de néctar y pulpa.

\begin{tabular}{lcrcc}
\hline & Concentración & Tiempo & Temperatura & $\Delta \mathbf{E}$ \\
\hline PULPA & 0,075 & 75,0 & 35 & 5,8 \\
NECTAR & 0,100 & 100,5 & 35 & 6,4 \\
NECTAR & 0,100 & 95,0 & 40 & 8,3 \\
\hline \multicolumn{2}{l}{$\mathrm{E}=$ Cambio de color } & & &
\end{tabular}




\section{CONCLUSIONES}

La aplicación de enzimas pectoliticas en la elaboración de pulpa néctar a partir de frutas, es de gran utilidad debido a que se obtienen productos con viscosidades similares a los productos existentes sin necesidad de agregar agua para tal fin. Para obtener una pulpa con una viscosidad aproximada de $477 \mathrm{mPa}^{*} \mathrm{~s}$ es necesario realizar un tratamiento a $35^{\circ} \mathrm{C}$ por 75 minutos y con una concentración de enzima de $0,075 \%$ y para la obtención de un producto tipo néctar con una viscosidad de aproximadamente $54 \mathrm{mPa}^{*} \mathrm{~s}$ es necesario realizar un tratamiento a $35^{\circ} \mathrm{C}$ por 100,5 minutos y con un concentración de enzima de $0,1 \%$.

Se tiene en cuenta inicialmente la comparación de las tres variables frente a la viscosidad y no al color debido a que no es el objetivo principal de este trabajo proteger el color de los productos obtenidos, aunque es una variable que afecta visualmente la calidad del producto, por el contrario la viscosidad es la variable de respuesta más importante debido que dependiendo del valor de este parámetro, es el producto procesado obtenido, es decir si el producto se clasifica como un néctar, una pulpa o un jugo.

\section{REFERENCIAS}

Alkorta, I., Garbisu, C., Llama, M. J. y Serra, J. 1998. Industrial applications of pectic enzymes: a review. Process Biochemistry 33(1):21-28.

Bhattacharya, S. and Rastogi, N. K. 1998. Rheological properties of enzyme- treated mango pulp. Journal of Food Engineering 36(3):249-262.

Bon, J., Vaquiro, H., Benedito, J. and TelisRomero, J. 2010. Thermophysical properties of mango pulp (Mangifera indica L. cv. Tommy Atkins). Journal of Food Engineering 97(4):563-568.

Ceci, L. y Lozano, J. 1998. Determination of enzymatic activities of commercial pectinases for the clarification of apple juice. Food Chemistry 61(1-2):237-241.

Demir, N., Acar, J., Sarioglu, K. and Mutlu, M. 2001. The use of commercial pectinase in fruit juice industry. Part 3: Immobilized pectinase for mash treatment. Journal of Food Engineering 47(4):275-280.

Missang, C., Massiot, P., Baron, A. and Drilleau, J.F. 1993. Effect of oxidative browning of apple pulp on the chemical and enzymatic extraction of cell wall polysaccharides. Carbohydrate Polymers 20(2):131-138.

Grassin, C. and Fauquembergue, P. 1996. Application of pectinases in beverages, In: J Visser and A.G.J. Voragen, Editor(s), Progress in Biotechnology, Elsevier, Pectins and Pectinases. Proceedings of an International Symposium 14:453-462.

lagher, F., Reicher, F. and Ganter, J.L. 2002. Structural and rheological properties of polysaccharides from mango (Mangifera indica L.) pulp. International Journal of Biological Macromolecules 31(1-3):9-17. 
Karunasawat, K. and Anprung, P. 2010. Effec of Depolymerized Mango Pulp as a Stabilizer in, Oil-in-Water Emulsion Containing Sodium Caseinate. Food and Bioproducts Processing, In Press, 88, (23):202-208.

Kashyap, D. R., Vohra, P. K., Chopra, S. and Rewari. R. 2001. Applications of pectinases in the commercial sector: a review. Bioresource Technology $77(3): 215-227$.

Liew, Abdullah, A.G., Sulaiman, N.M., Aroua, M.K. y Megat, M.J. 2007. Response surface optimization of conditions for clarification of carambola fruit juice using a commercial enzyme. Journal of Food Engineering 81(1):65-71.

Pelegrine, D.H., Silva, F. C. and Gasparetto, C. A. 2002. Rheological Behavior of Pineapple and Mango Pulps. Lebensmittel-Wissenschaft undTechnologie 35(8):645-648.

Pretel, M.T., Lozano, P., Riquelme, F. and Romojaro, F. 1997. Pectic enzymes in fresh fruit processing: optimization of enzymic peeling of oranges. Process Biochemistry 32(1)43-49.
Quaglia G B, Gennaro L, (2003). ENZYMES

I Uses in Food Processing, In: Benjamin Caballero, Editor(s)-in-Chief, Encyclopedia of Food Sciences and Nutrition, Academic Press, Oxford, 2125-2139.

Rai, P., Majumdar, G.C., and Dasgupta, S. De S. 2004. Optimizing pectinase usage in pretreatment of mosambi juice for clarification by response surface methodology. Journal of Food Engineering 64(3):397-403.

Sarioglu, K., Demir, N., Acar, J. and Mutlu, M. 2001. The use of commercial pectinase in the fruitjuice industry, part 2: Determination of the kinetic behaviour of immobilized commercial pectinase. Journal of Food Engineering 47(4):271-274.

Sreenath, H.K., Sudarshana, Krishna, K.R. and Santhanam, K. 1995. Enzymatic liquefaction of some varieties of mango pulp. LWT - Food Science and Technology 28(2):196-200.

Valente, M. y Ferrandis, J.I. 2003. Evaluation of textural properties of mango tissue by a near-field acoustic method. Postharvest Biology and Technology 29(2):219-228. 